\title{
Effects of Empathy on the Evolutionary Dynamics of Fairness in Group-Structured Systems
}

\author{
Yanling Zhang $\mathbb{D}^{1,2}$ Jian Liu $\mathbb{D}^{1},{ }^{1}$ and Aming $\mathrm{Li} \mathbb{D}^{3}$ \\ ${ }^{1}$ Key Laboratory of Knowledge Automation for Industrial Processes of Ministry of Education, \\ School of Automation and Electrical Engineering, University of Science and Technology Beijing, Beijing 100083, China \\ ${ }^{2}$ Institute of Artificial Intelligence, University of Science and Technology Beijing, Beijing 100083, China \\ ${ }^{3}$ Department of Zoology, University of Oxford, Oxford OX1 3PS, UK \\ Correspondence should be addressed to Aming Li; aming.li@zoo.ox.ac.uk
}

Received 5 March 2019; Revised 13 August 2019; Accepted 4 September 2019; Published 18 November 2019

Academic Editor: Lucas Lacasa

Copyright ( 2019 Yanling Zhang et al. This is an open access article distributed under the Creative Commons Attribution License, which permits unrestricted use, distribution, and reproduction in any medium, provided the original work is properly cited.

\begin{abstract}
The ultimatum game has been a prominent paradigm in studying the evolution of fairness. It predicts that responders should accept any nonzero offer and proposers should offer the smallest possible amount according to orthodox game theory. However, the prediction strongly contradicts with the experimental behaviors where the mean offer typically ranges from 0.3 to 0.5 and the mean demand tends to lie between 0.2 and 0.35 . To explain the evolution of such fair behaviors, here we introduce empathy in a mutation-selection process with group structure and find that our results quantitatively reproduce the experimental behaviors at low randomness with intermediate empathy or relatively high randomness with small empathy. Moreover, we show that with low randomness more empathy leads to a fairer outcome with a higher mean offer and demand. Counterintuitively, more empathy corresponds to a lower mean offer together with a higher mean demand for relatively high randomness. Finally, we analytically provide the mean offer and demand under both weak and strong intensities of selection when the largest or smallest level of empathy is introduced. Our study provides systematic insights into the evolutionary origin of fairness in a mutation-selection process with empathetic strategies and group structure.
\end{abstract}

\section{Introduction}

In the canonical ultimatum game, one player proposes a division of a sum of money between himself/herself and a second player, who might accept or reject the proposal. If the proposal is accepted, the sum is shared accordingly; if not, both players remain empty-handed. On the assumption of orthodox game theory, the responder should accept any nonzero offers and the proposer should offer the smallest possible amount. However, human behavioral experiments have shown a different reality. The mean offer typically ranges from 0.3 to 0.5 , and the mean demand tends to lie between 0.2 and $0.35[1,2]$. Apart from behavioral experiments $[3,4]$, the evolution of fairness has also been studied using a great diversity of models under the framework of game theory and evolutionary game theory [5].

Evolutionary game dynamics focuses on the evolution of fairness among selfish and interactive individuals [6-14], where strategies with higher fitness are more likely to spread among individuals. Under this evolutionary framework, the conditions under which the fair strategy is favored by natural selection have been attracting considerable interest. In wellmixed populations without invoking additional mechanisms, natural selection itself can lead to the rational selfinterest strategy, where agents offer the smallest amount and accept any nonzero offers $[15,16]$. Considering this, researchers have investigated a large number of factors to explain how the fair strategy can be favored in the twoperson ultimatum game [15-34]. These factors are reputation [15], empathy [16-20], alternating role [21, 22], randomness [23, 24], spite [25, 26], altering the stake size [27], spatial population structure $[18,28-34]$, and so on. Particularly, some models comprise different factors, for example, the effects of empathy [18], adaptive role switching [21, 22], random allocation [24], and migration [34] have been studied in spatially structured populations. 
Empathy, meaning that individuals only make offers that they would themselves be ready to accept, has attracted much attention in modeling human behavior. When all individuals are assumed to play empathetic strategies, fair offers can be favored by natural selection in well-mixed populations [17]. Under the same or very similar assumption, empathetic players also result in the evolution of fairness in regular graphs [18] and other complex networks [19]. Page and Nowak have found that in well-mixed populations, allowing a small proportion of the population to play empathetic strategies is enough to favor the evolution of fairness [20]. However, if natural selection acts upon the proportion of empathetic strategies, they also have shown that empathy is not selected by natural selection. A recent literature has found when individuals are restricted to interact with a fraction of the total population, e.g., group structure, empathetic individuals can survive in the population [35]. The survival of empathy itself is the premise of studying the effects of empathy on fairness, which is also an important motivation for adding group structure in this paper.

Group structure is ubiquitous in real society because individuals are often distributed to form numerous groups instead of interacting equally in well-mixed populations. For example, the group can be understood as an island in population genetics or a particular organization in human society, as shown in [35-37]. Considering individuals in real life may be exchanged between nearby groups [38] and the advantages of temporal networks over their static counterparts [39-42], we incorporate migration in the model as well and allow the interactive network to change with time. Our model is stimulated by the classical stepping-stone model, which has been extensively adopted by mathematicians and biologists (see [43] and its references). Indeed, we consider the case where each node represents a group with arbitrary number of individuals, and global update is also used in our model. However, previous studies on empathy in spatial populations merely focused on games on graphs, where each node represents an individual and local update is adopted [18, 19]. And they assumed that all or almost all individuals are empathetic. It is still open whether more empathy will lead to a fairer outcome in a spatial population. Therefore, we allow an arbitrary level of empathy initially introduced in populations with group structure. Note that we also find that the group structure has obvious disadvantages in promoting fairness compared with spatial populations in $[18,19]$. Furthermore, beyond the group structure, our another goal is studying the effects of empathy on the evolution of fairness.

In this paper, we introduce empathy by initially allowing a proportion $\alpha$ of the population to play empathetic strategies and $\alpha$ represents the level of empathy. It appears that the single-group case of our model degenerates to the one by Page and Nowak [20]. Yet, this does not necessarily mean our model is the same as that in [20], or just a simple extension from [20]. In fact, these two models have two significant differences: (1) strategy and empathy evolve according to the mutation-selection process in our model but evolve by the modified adaptive dynamics in their model.
(2) Our model adopts global mutation (i.e., mutants can be quite different from the parent), but their model is limited to local mutation (i.e., mutants have to be close to the parent). This is essentially important as it has been shown that the mutation mode strongly influences the evolutionary results of asymmetric binary games [44]. Moreover, in [20], the mean offer and demand are both shown close to 0.5 , which is clearly not in accordance with the experimental evidence. Importantly, by considering global mutation and the intensity of selection, we find the conditions under which the experimental behaviors are reproduced quantitatively.

Indeed, it is not realistic to assume that individuals always have enough knowledge on how to obtain higher payoffs or others strategies. As clearly reported in $[45,46]$, cultural evolution may occur with high probabilities of strategy exploration, analogous to mutation. The effects of high mutation probabilities on evolutionary dynamics also have received much attention [23, 47]. To explain the experimental behaviors and further to explore the factors favoring fairness, we make a comprehensive analysis by considering all possible values of mutation probability and level of empathy. Note that, in terms of the parametric space, previous studies mainly focus on low-mutation probabilities (probability is from 0.001 to 0.01 ). Furthermore, we also investigate how the intensity of selection and group number influences the evolution of fairness. Under both weak and strong intensities of selection, we analytically provide the mean offer and demand for the largest and smallest level of empathy.

\section{Model}

2.1. Ultimatum Game. In the ultimatum game with a proposer and a responder, the proposer suggests how to split a fixed sum (say 1) and the responder decides whether or not to accept the proposal. When the responder accepts it, the offer is split accordingly; otherwise, both individuals get nothing. Each individual has a strategy denoted by a vector $(p, q)$ where $p \in[0,1]$ is the amount offered when he acts as a proposer, and $q \in[0,1]$ is the minimum amount demanded when he acts as responder. In each pairwise interaction, either individual acts once as a proposer and once as a responder. An offer $p_{1}$ is accepted by a responder with the minimum demand $q_{2}$ if and only if $p_{1} \geq q_{2}$. Therefore, in a pairwise interaction, the payoff for an individual using the strategy $\left(p_{1}, q_{1}\right)$ against another individual using the strategy $\left(p_{2}, q_{2}\right)$ is given by

$$
a\left(\left(p_{1}, q_{1}\right),\left(p_{2}, q_{2}\right)\right)= \begin{cases}0, & p_{1}<q_{2}, p_{2}<q_{1}, \\ p_{2}, & p_{1}<q_{2}, p_{2} \geq q_{1}, \\ 1-p_{1}, & p_{1} \geq q_{2}, p_{2}<q_{1}, \\ 1-p_{1}+p_{2}, & p_{1} \geq q_{2}, p_{2} \geq q_{1} .\end{cases}
$$

2.2. Evolutionary Dynamics. If a strategy $(p, q)$ satisfies $p=q$, i.e., the offer that an individual makes is equal to the minimum offer that he prepares to accept, the strategy is 
called empathetic strategy. If $p$ and $q$ of a strategy are independent of each other, the strategy is called independent strategy. Consider a population with $N$ individuals in which each individual belongs to one of the $M$ groups. Initially, each individual adopts an empathetic strategy with probability $\alpha \in[0,1]$, where the value of $p=q$ follows the uniform distribution over $[0,1]$; otherwise, with probability $1-\alpha$, he adopts an independent strategy, where the values of $p$ and $q$ follow the uniform distribution over $[0,1]$. Each individual interacts with other individuals in the same group, and in each pairwise interaction either individual acts once as a proposer and once as a responder. After an individual $i$ interacts with all other individuals in the same group, he receives the total payoff $P_{i}$. The number of interactions that an individual participates in depends on the size of the group the individual is located in, and the number of games is twice that of interactions. For example, 20 individuals are located in the first group, and then the number of games each individual plays in this group is $20 \times 19=380$. The fitness of the individual $i$ is defined as $1+\omega P_{i}$, where $\omega$ is called the intensity of selection [48]. Two extreme cases $\omega \longrightarrow 0$ and $\omega \longrightarrow 1$ correspond to weak selection and strong selection, respectively.

The strategy update of all individuals follows the frequency-dependent Moran process [48]. As shown in Figure 1(a), one individual is randomly chosen to "die" and another individual is chosen to "reproduce" an offspring proportional to his fitness at each generation. The newborn offspring adopts his parent's strategy with probability $1-u$; otherwise, he mutates to a random empathetic strategy with probability $\alpha$ and a random independent strategy with probability $1-\alpha$. The Moran process is a natural scenario for genetic evolution and also translates into social settings. A random individual resolves to update his strategy; subsequently, he adopts another individual's strategy, who is chosen proportional to his fitness. It is noteworthy that the proportion of empathetic strategies fluctuates around the level of empathy $\alpha$ during the evolutionary process, which is different from the previous literature [20], where the proportion is maintained during the evolutionary process by assuming that each individual offers his $q$-value with probability $\alpha$. Particularly, all individuals adopt independent strategies for the smallest level of empathy $\alpha=0$ and empathetic strategies for the largest level of empathy $\alpha=1$ during the whole evolutionary process.

2.3. Migration Range $r$. Initially, $N$ individuals are randomly distributed to $M$ groups. Migration is a fundamental feature in humans and animals. Motivated by the classical steppingstone model (see [43] and the references therein) and following the preceding studies [49-51], we choose a kind of diverse migration-random migration, which incorporates many possible ways of migration and assumes that only the newborn individual migrates to one new group with probability $v$; otherwise, he stays in his parent's group.

We adopt the simplest assumption that the groups are located on a circle, which describes the relative physical distances between any two groups [43]. On the one hand, the simplest structure allows us to extract the effects of other parameters on fairness, such as the level of empathy. Indeed, the structure of circle is generally employed to investigate rich migration strategies in structured populations, and a typical example is the homogeneous migration pattern intensively discussed in the population genetics [43]. On the other hand, although our analytical results are under weak selection, we have checked that they hold true as well for more complex structures, such as two-dimensional or multiple-dimensional lattices.

We make use of graph theory to illustrate the migration strategy, where each node is a group and the edges are potential single-step migration paths. We focus on homogeneous graphs in the sense that they look the same from every node, and migration strategies of this sort have been intensively discussed in the population genetics (see [52] and its references). To pay more attention to the effects of other parameters on fairness, we investigate a type of homogeneous migration patterns, which is described by the migration range $r$ in Figure 1(b). The migration range $r$ means that an individual can equally migrate to groups that are within $r$ steps far from his parent's group. Note that here different values of $r$ also generate another angle of the interaction structure, which can exhibit enormous configurations.

\section{Results}

3.1. Simulation Results. We first perform agent-based simulations by varying the intensity of selection $\omega$, the level of empathy $\alpha$, and the group number $M$. For each set of simulation parameters, we determine the mean offer and demand, which are the time-averaged values of $p$ and $q$ over the whole population, respectively. Here, the mean offer $p$ and demand $q$ are averaged over $5 \times 10^{7}$ generations for the mutation probability $u \geq 0.1$ and $5 \times 10^{8}$ generations for $u<0.1$, which ensures that the population has reached the stationary state. Specifically, we have exported a set of $p$-value and $q$-value after every $10^{7}$ generations and have validated that the changes over the last five sets of values fluctuate below 0.001 . The standard deviations of our simulation results are very small, and the difference generated by different random realizations can be negligible (shown in Table 1 of Supplementary Information). Therefore, we do not show the variances of the following simulation results. Our evolutionary process is a Markov chain due to nonzero mutation probabilities, and our simulation results are obtained based on the stationary distribution which is identical to the limiting distribution according to the ergodic theorem and the limiting theorem. Therefore, our results are independent of the initialization (shown in Table 2 of Supplementary Information) and we adopt the uniform initialization.

When the intensity of selection $\omega$ is very small $(\omega \approx 0)$, neutral drift dominates the evolutionary dynamics, indicating that the mean offer and demand are close to 0.5 (Figures 2(a)-2(d)). As $\omega$ increases but is still small, both the mean offer and demand decrease significantly with $\omega$ (Figure 2). When the level of empathy $\alpha$ is small, the decreasing trends mainly depend on the evolution of 


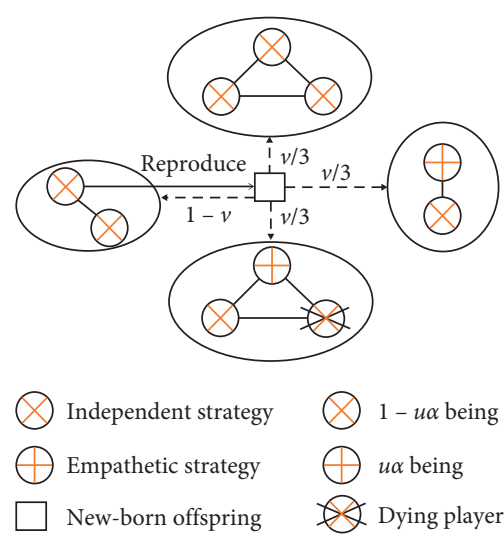

(a)
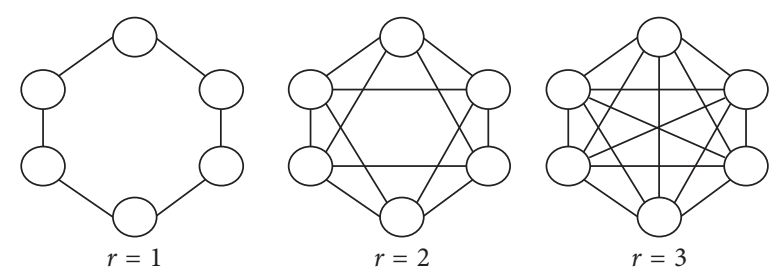

(b)

Figure 1: Model schematic. (a) Evolutionary dynamics with global migration. Ten individuals (represented by nodes, $N=10$ ), eight of whom (nodes with $\times$ ) play independent strategies and the remainder (nodes with + ) play empathetic strategies $(p=q$ ), are distributed to four groups (shaded by blue, $M=4$ ). At a given generation, an individual with an independent strategy is chosen to reproduce one offspring (empty square) and an individual (node under cross) is chosen to die. The newborn offspring adopts independent strategies with probability $1-u \alpha$ and empathetic strategies with $u \alpha$. The offspring stays in his parent's group with probability $1-v$, and he migrates to another group with $v / 3$. (b) Illustration of the migration range $r$. Each node (white circle) and each edge represent a group and a potential migration path, respectively. For example, when $r=1$, an individual equally migrates to two groups that are the direct neighbors of his parent's group.

independent strategies. According to equation (1), a lower offer has a payoff advantage over a higher offer for independent strategies, i.e., $a((p, *),(p+\varepsilon, *)) \geq a((p+\varepsilon, *)$, $(p, *))$ with $\varepsilon>0$. Moreover, the increase of $\omega$ significantly enlarges the payoff advantage of lower offers over higher offers. Therefore, we have the significant decrease of the mean offer with $\omega$. The decrease of the mean offer drives the mean demand to decrease accordingly in order to avoid rejection. When $\alpha$ is large, the decreasing trends mainly depend on the evolution of empathetic strategies. According to equation (1), for empathetic strategies, the strategy with $p<0.5$ has a payoff advantage over the strategy with $p>0.5$; i.e., the payoff of $p=0.5-\varepsilon(\varepsilon>0)$ is more than that of $p=0.5+\varepsilon$. The initial increase of $\omega$ enlarges the payoff advantage of $p<0.5$ over $p>0.5$, and it leads to the significant decrease of the mean offer and demand.

When the intensity of selection $\omega$ becomes sufficiently large and further increases, the mean offer or demand remains around nonzero constants (Figure 2). The reason for the existence of the nonzero constant is as follows. The numerator and the denominator of the probability that an individual reproduces an offspring linearly depend on $\omega$. When $\omega$ is sufficiently large, it can be simultaneously eliminated from the numerator and the denominator. Accordingly, the payoff advantage of lower offers over higher offers or the payoff advantage of $p<0.5$ over $p>0.5$ cannot be enlarged by increasing $\omega$. Consequently, the mean offer or demand cannot change with $\omega$ and remains around nonzero constants.

When the intensity of selection $\omega$ is very small or the mutation probability $u$ is very high (Figure S1 of Supplementary Information), the mean offer and demand change very little with the level of empathy $\alpha$ because neutral drift or mutation dominates the evolutionary dynamics. For very low-mutation probabilities and large intensities of selection, generating low randomness, the mean offer and demand both increase and get closer to the fair ones with the level of empathy (Figures 3(c) and 3(d)). It implies that with low randomness, more empathy leads to a fairer outcome with a higher mean offer and demand. For small intensities of selection or for large intensities of selection and intermediate mutation probabilities, corresponding to relatively high randomness, the mean offer decreases and yet the mean demand increases with the level of empathy (Figure 3). It implies that with relatively high randomness, more empathy results in a lower mean offer and a higher mean demand.

The results can be intuitively understood by comparing two extreme cases $\alpha=0$ and $\alpha=1$. For the smallest level of empathy $\alpha=0$, we have known that lower offers have the payoff advantage over higher offers. For the largest level of empathy $\alpha=1, p<0.5$ closer to 0.5 has the payoff advantage over $p<0.5$ further away 0.5 or $p>0.5$ according to equation (1); i.e., a strategy with $p<0.5$ has a higher payoff than that with $p-\varepsilon(\varepsilon>0)$ or a strategy with $p>0.5$. Therefore, a lower offer $p$ and a lower demand $q$ will be favored by natural selection for $\alpha=0$ and yet the offer $p$ closer to 0.5 will be favored for $\alpha=1$. For very low-mutation probabilities and large intensities of selection, natural selection plays a major role in strategy evolution, and thus the mean offer and demand for $\alpha=0$ are less than those for $\alpha=1$. For intermediate mutation probabilities and large intensities of selection, mutation plays a major role in strategy evolution. Strategies with high offers or high demands are frequently introduced in the evolutionary process for $\alpha=0$, but only strategies with high offers and high demands are frequently introduced for $\alpha=1$. However, only strategies with high offers and low demands can achieve enough payoffs to survive in the population. Therefore, the mean offer for $\alpha=0$ is more than the one for $\alpha=1$, and yet the mean demand for $\alpha=0$ is less than the one for $\alpha=1$. The 

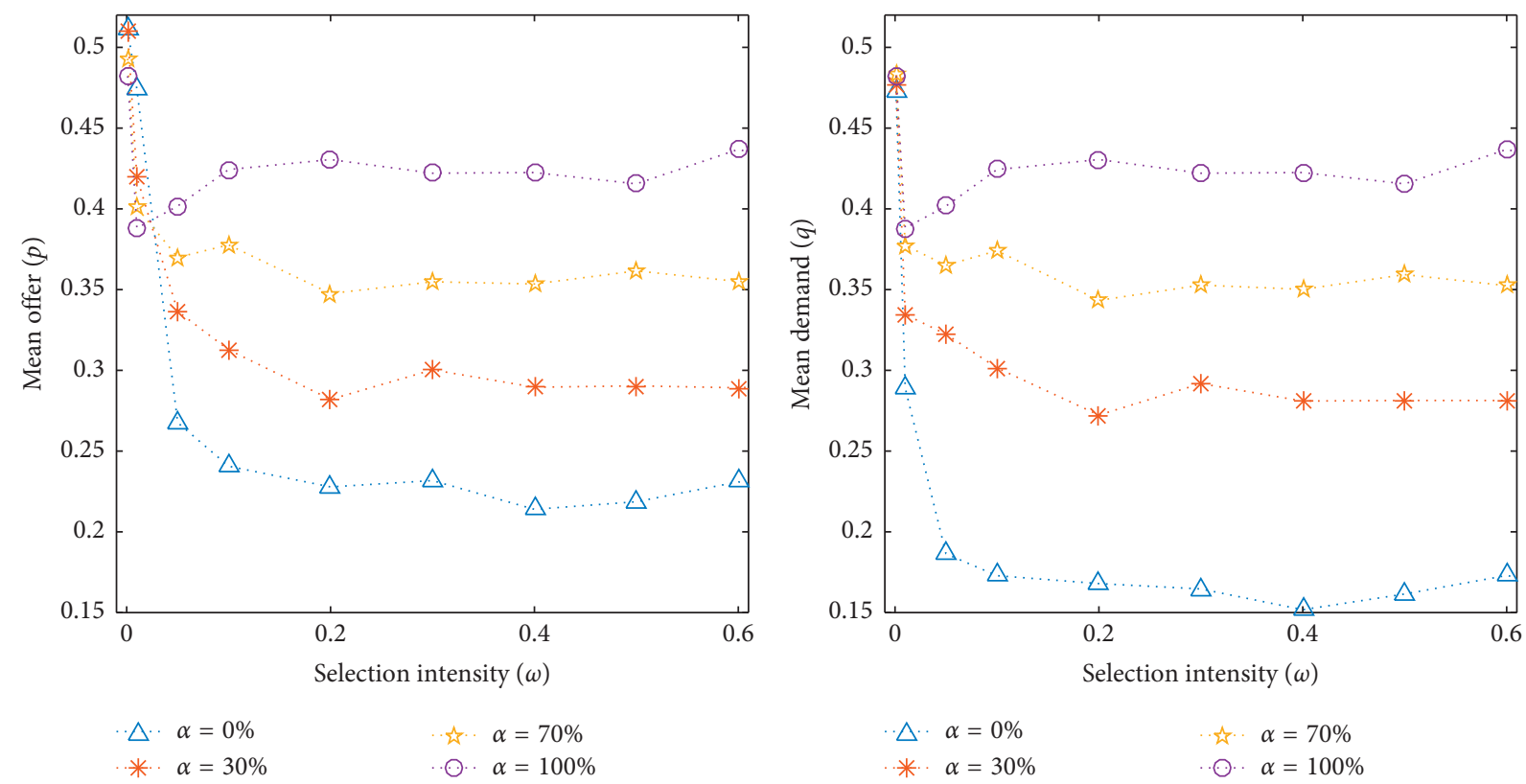

(a)

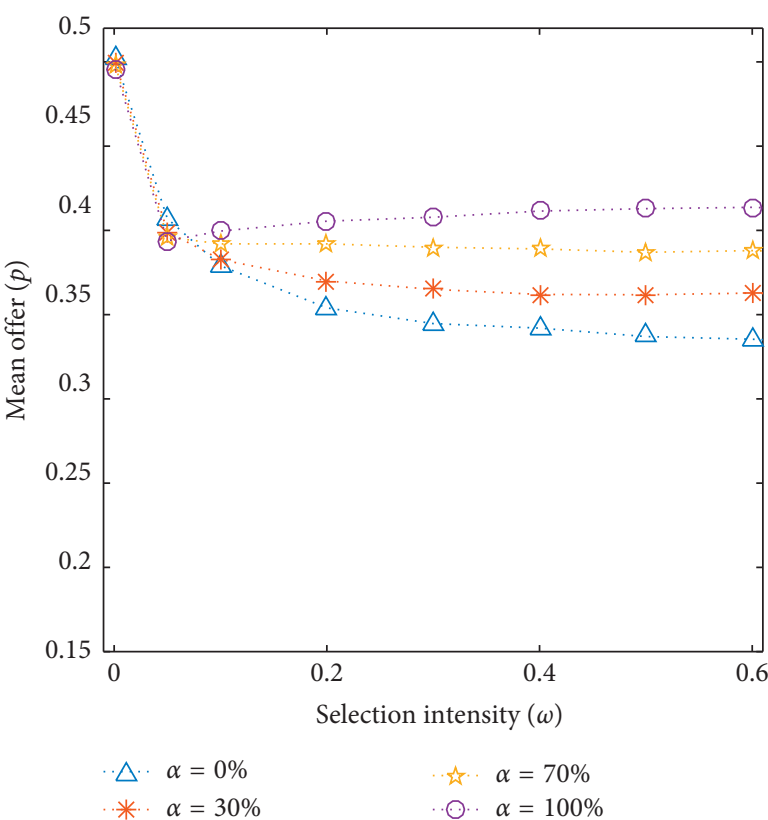

(c)

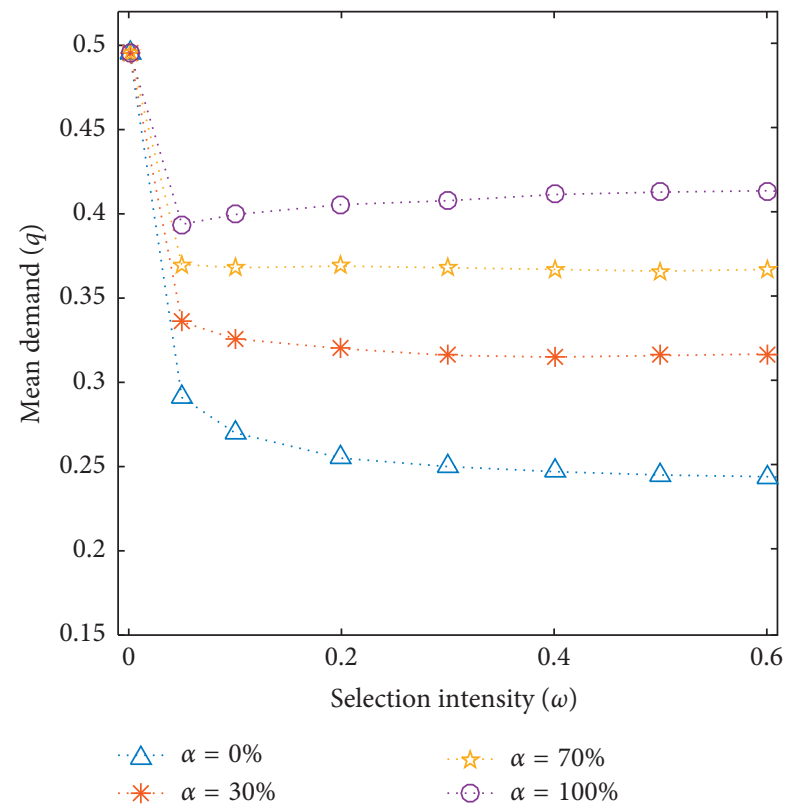

(d)

FIGURE 2: The changing trends of the mean offer $p$ and demand $q$ with the intensity of selection $\omega . p$ and $q$ significantly decrease when $\omega$ increases from 0 . They remain around nonzero constants when $\omega$ is sufficiently large. Parameters: $N=50, M=9, r=1$, and $v=0.1$, with $u=0.005$ in $(\mathrm{a}, \mathrm{b})$ and $u=0.1$ in $(\mathrm{c}, \mathrm{d})$.

same result also holds true for small intensities of selection, where neutral drift plays a major role in strategy evolution. This is because strategies with higher offers and lower demands can obtain enough payoffs to survive for $\alpha=0$ and strategies with $p<0.5$ further away from 0.5 can for $\alpha=1$, since they reach most deals with others.

When there is no migration (the migration probability $v=0$ ), the evolutionary result changes very little with the number of groups (Figures S2(a) and S2(d) of Supplementary Information), implying that the addition of group structure itself cannot promote the evolution of fairness compared with well-mixed structure. Because our model adopts accumulated payoffs and global updates, the group initially with most individuals is very likely to gamble more and more individuals and other groups become vacant. Finally, our group structure performs just similar to wellmixed structure when there is no migration.

As shown in Figures 4(a) and 4(b) (the level of empathy $\alpha=0$ ), when there is migration (the migration probability $v>0$ ), the mean offer and demand increase when the 


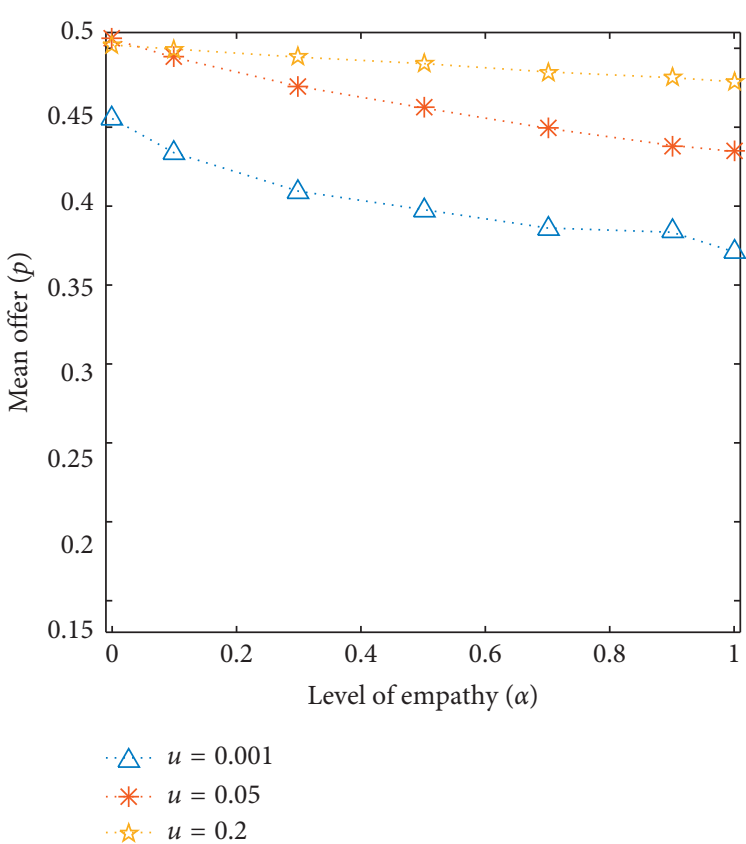

(a)

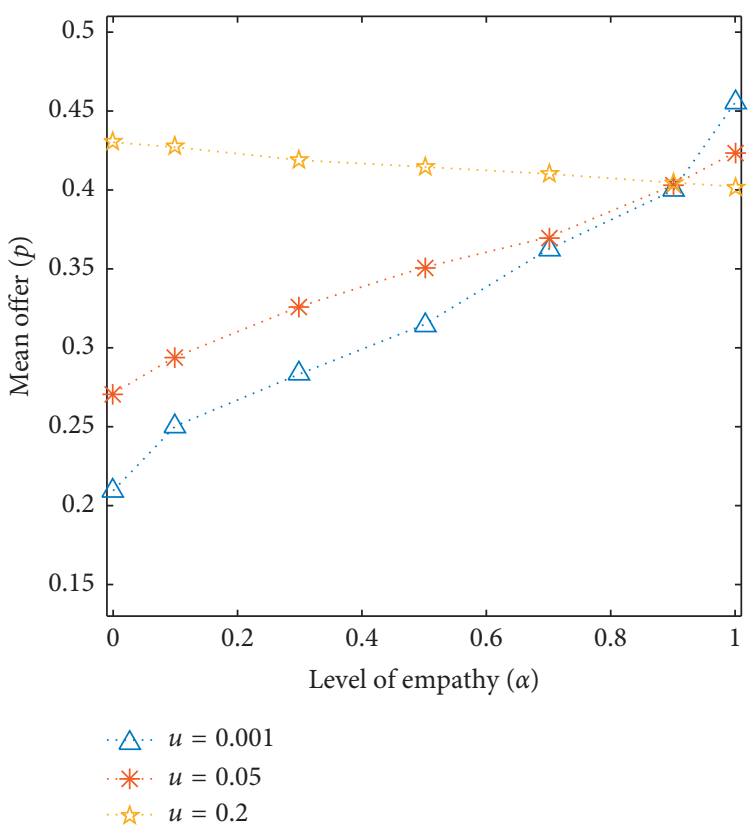

(c)

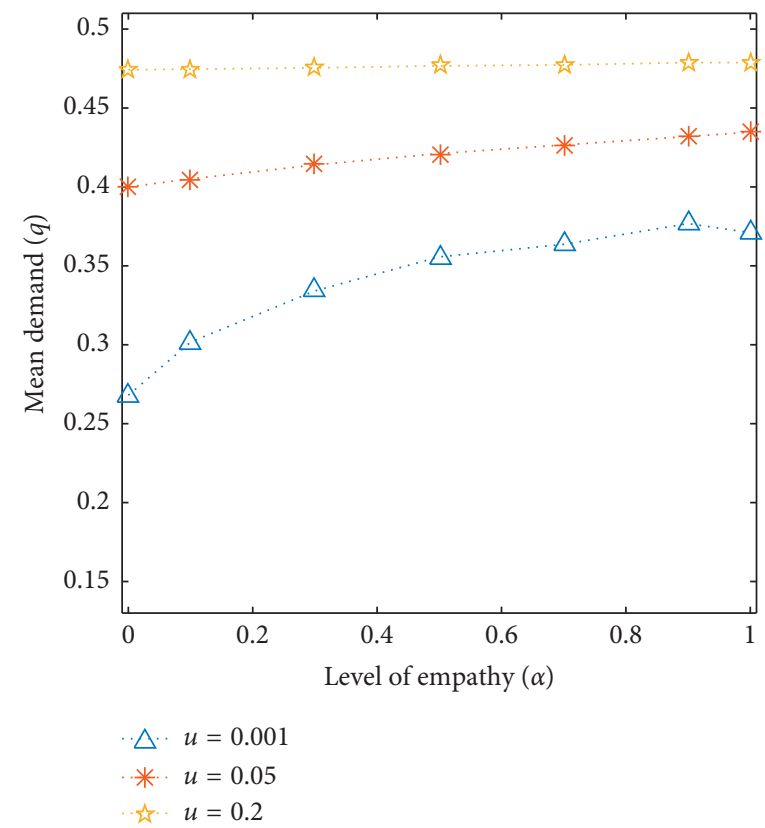

(b)

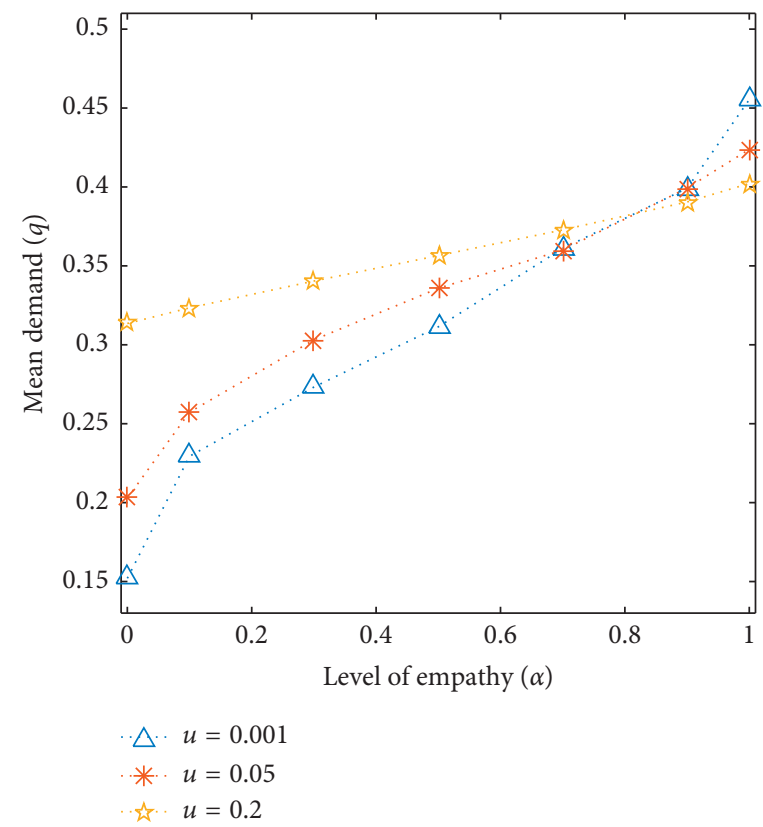

(d)

FIgURE 3: The changing trends of the mean offer $p$ and demand $q$ with the level of empathy $\alpha$. (a) $p$ decreases with $\alpha$ for all values of mutation probability $u$. (b, d) $q$ increases with $\alpha$ for all $u$. (c) $p$ increases with $\alpha$ for very low $u$ but decreases with $\alpha$ for intermediate $u$. Parameters: $N=50, M=9, r=1$, and $v=0.1$, with $\omega=0.01$ in (a,b) and $\omega=1$ in (c, d).

number of groups $M$ increases from 1, implying that the addition of group structure together with migration promotes the evolution of fairness compared with well-mixed structure. This is because multiple groups provide a higher mean offer or a higher mean demand with opportunities of obtaining enough payoffs to survive in the population. When $M$ further increases, the mean offer and demand are maintained around constants. In other words, the best promotion of fairness does not require too large $M$. Therefore, without loss of generality, we choose $M=7$ or $M=9$ in our simulations. The mean offer and demand increase with small migration ranges but remain around constants when the migration range $r$ is large (also see Figures S2(a) and S2(b) of Supplementary Information). To better understand whether other parameters promote fairness, we focus on $r=1$ in the above simulations. Motivated by the classical stepping-stone model (see [43] and the references therein) and following the preceding studies [49-51], we consider that only the newborn individual is allowed to 

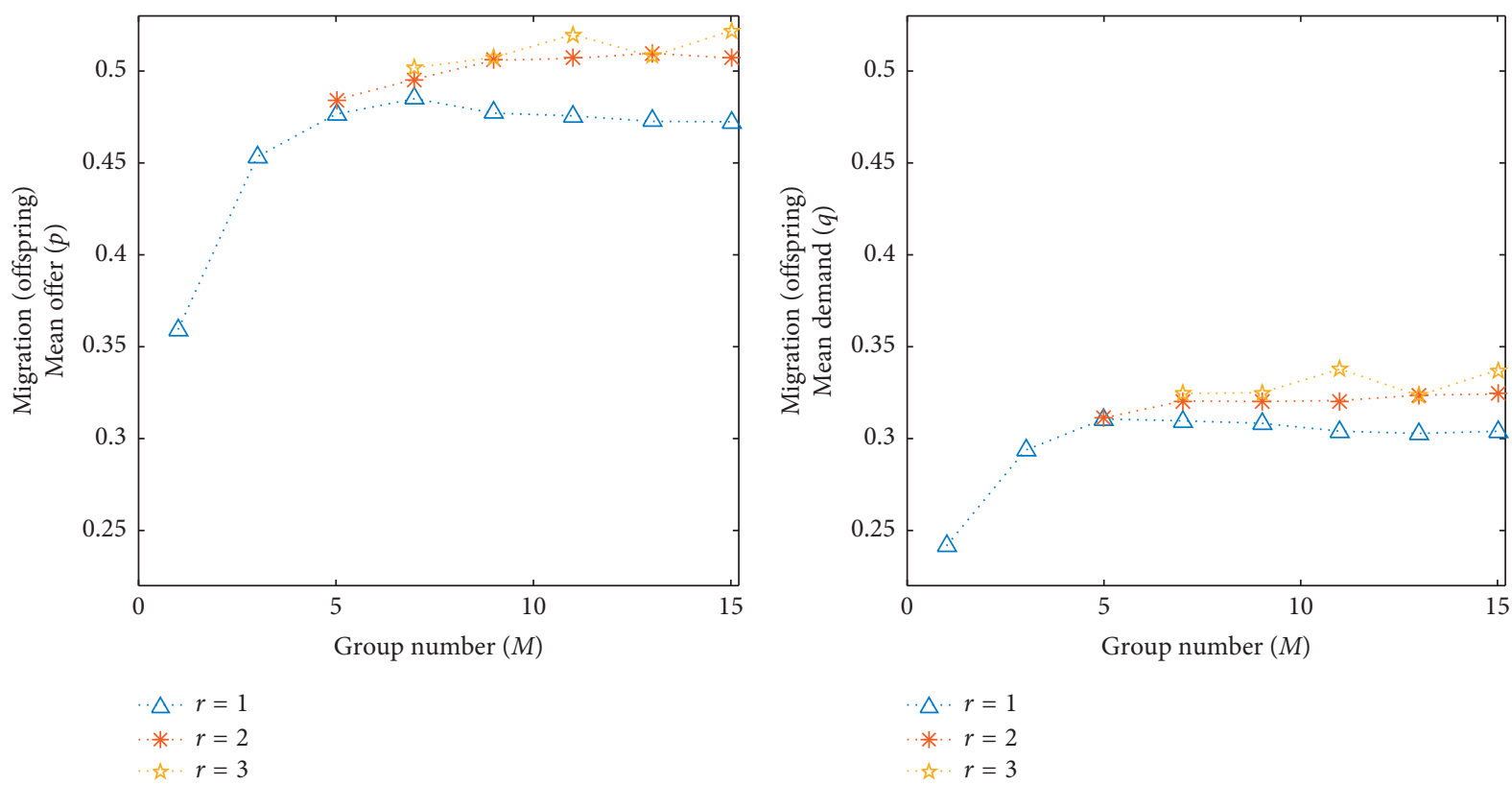

(a)

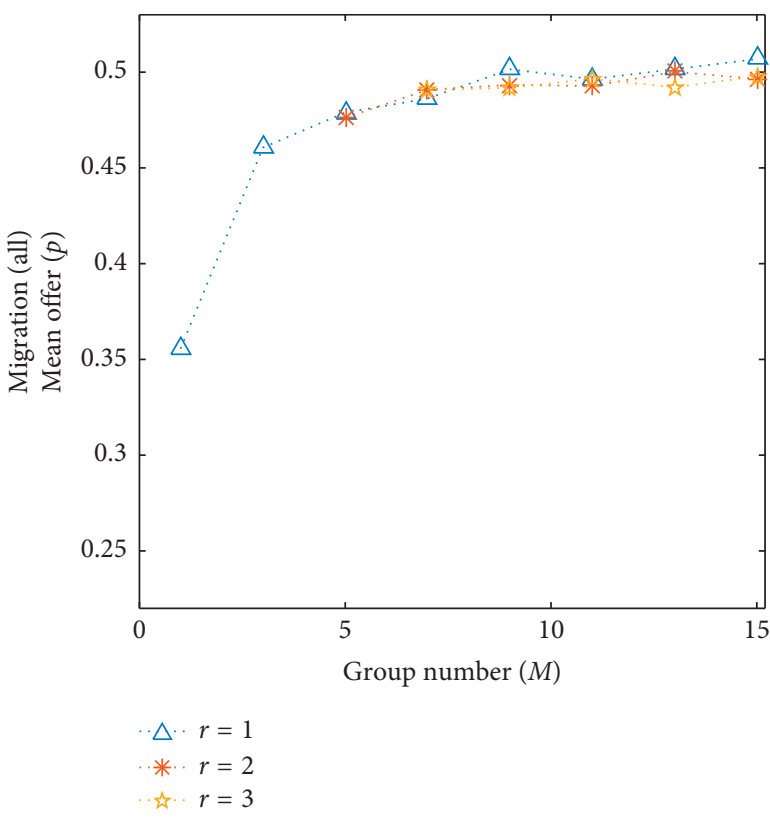

(c)

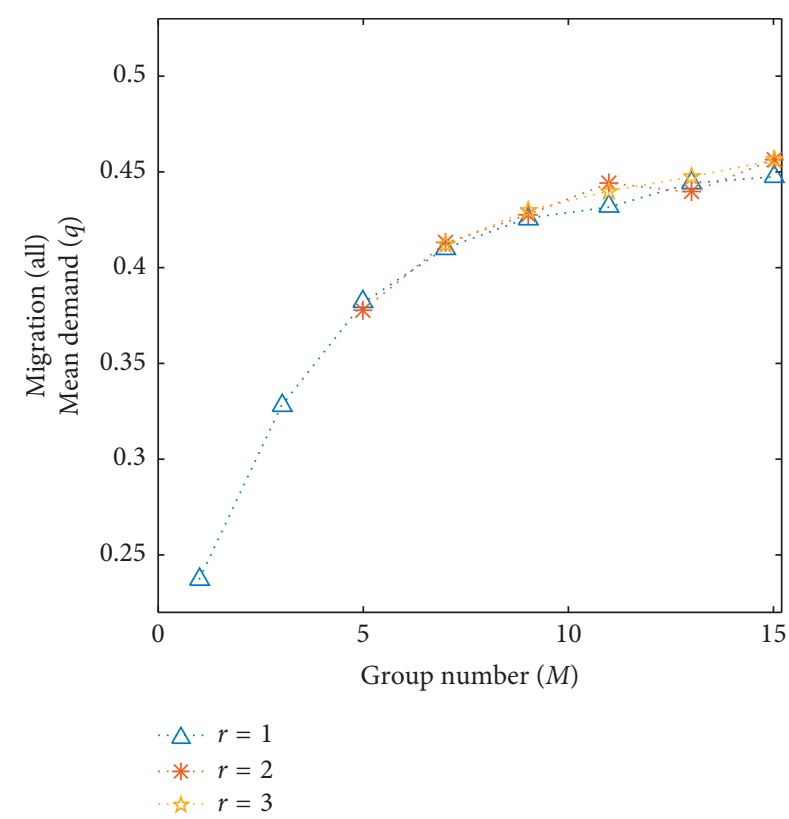

(d)

Figure 4: The changing trends of the mean offer $p$ and demand $q$ with the number of groups $(M)$ at the level of empathy $\alpha=0$. (a, b) The only offspring migrates with probability $v$ at each generation. (c, d) Each individual migrates with probability $v$ at each generation. Parameters: $N=50, v=0.1, u=0.01$, and $\omega=0.01$.

migrate at a generation. We also investigate the second case where all individuals migrate at a generation in Figures S2(a) and S2(b). We find that the mean offer and demand increase more with $M$ compared with the first case, and thus the promotion of fairness is enlarged in the second case. Different from the first case, the mean offer or demand does not change significantly with $r$.

3.2. Analytical Results. After presenting agent-based simulation results, we turn to theoretical calculations. It has been shown that, under weak selection, more empathy leads to a lower mean offer. Accordingly, we focus on the case with $\alpha=1$, where all players use continuous empathetic strategies. Here, a strategy can be described by a single parameter $p \in[0,1]$. The mean offer $p$ is calculated in Supplementary Information, and its analytical expression is as follows:

$$
p=\frac{1}{2}-\omega \frac{(1-u)(N-1)}{24 \mathrm{Mu}} \sum_{x=1}^{M}\left(\Psi_{1}(f(x ; r))-\Psi_{2}(f(x ; r))\right) \text {, }
$$




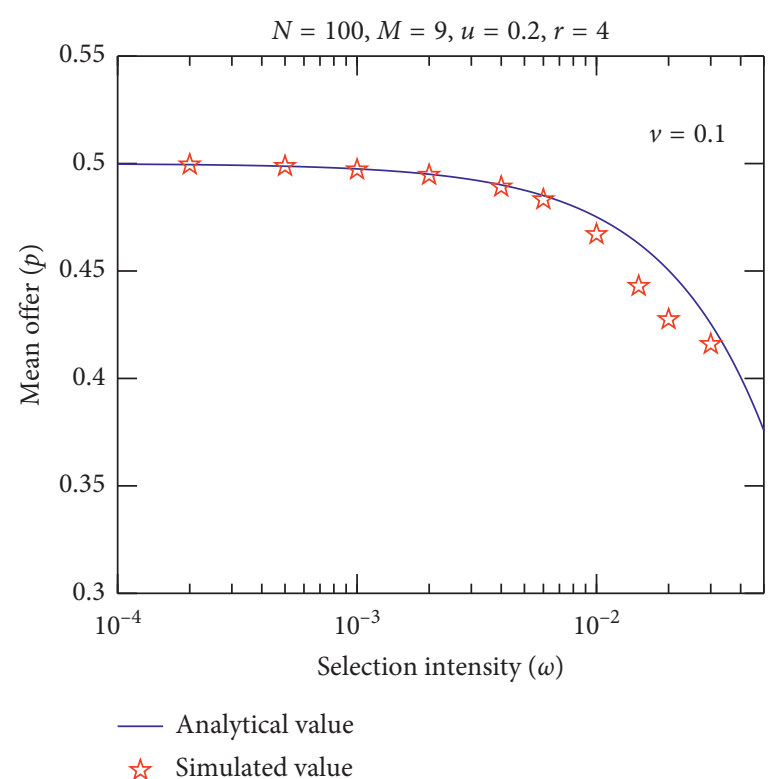

(a)

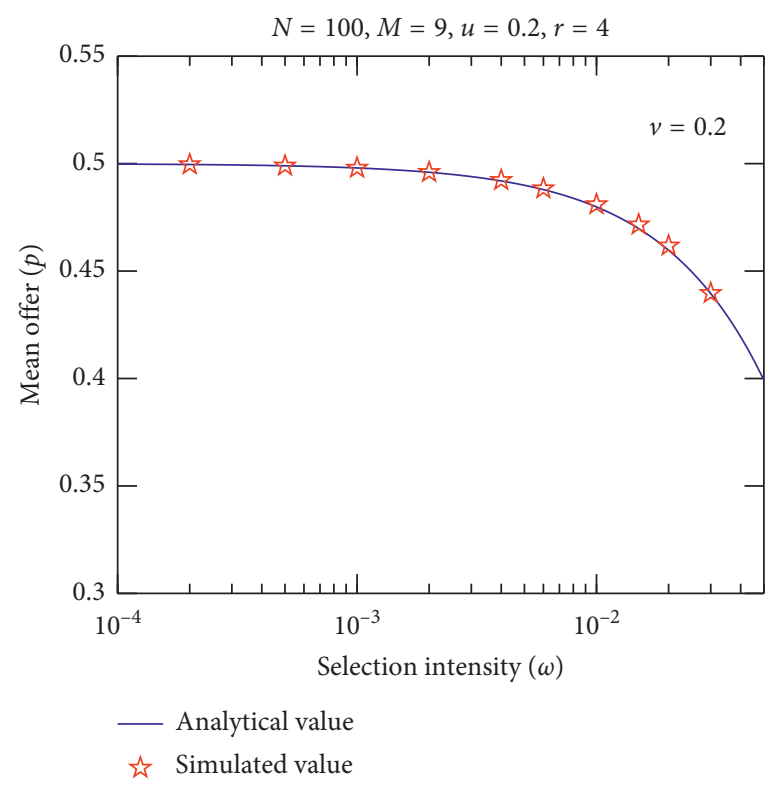

(c)

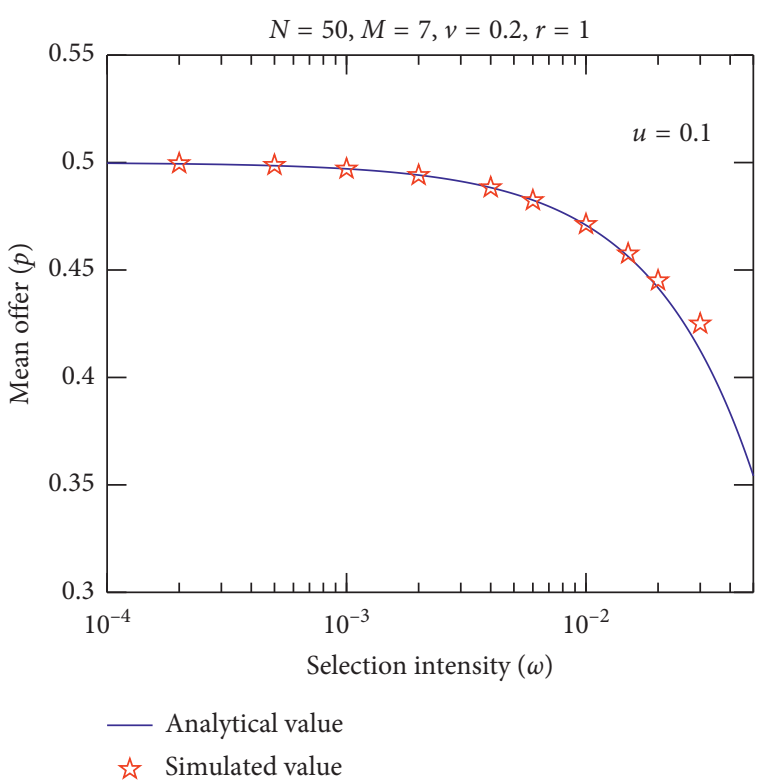

(b)

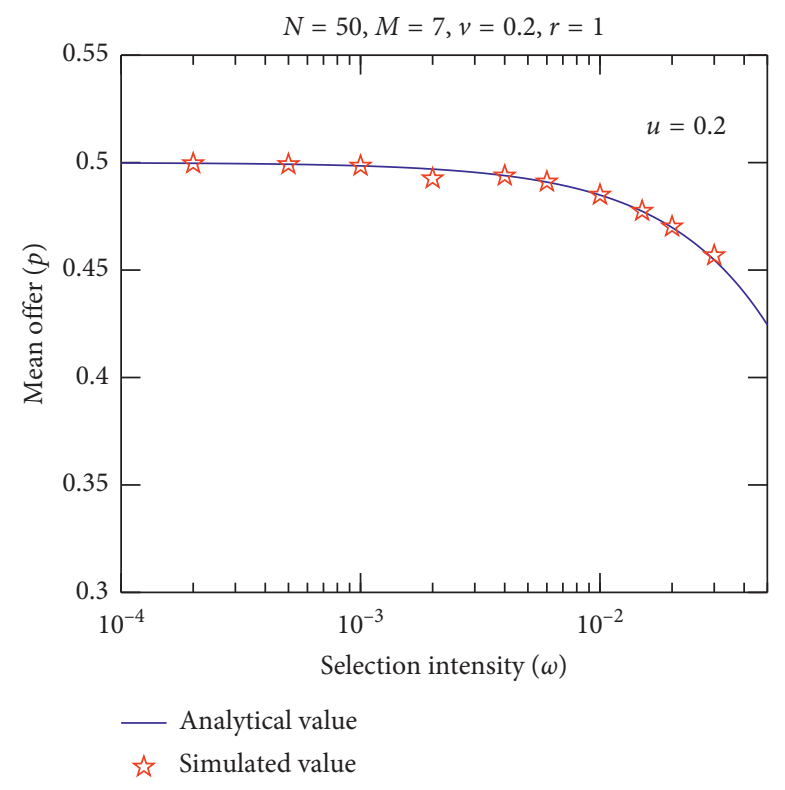

(d)

FIGURE 5: Under weak selection, comparison of theoretical results and numerical results. When the intensity of selection $\omega$ is small, our analytical calculations of the mean offer $p$ are in good agreement with the simulated values for different population sizes $(N)$, group numbers $(M)$, mutation probabilities $(u)$, migration probabilities $(v)$, and migration patterns $(r)$.

where $\quad \Psi_{1}(f)=(1-v(1-f)) /(1+(N-1) v(1-f))$, $\Psi_{2}(f)=((1-u)(1-v(1-f))) /(1+(N-1) u+(N-1)$ $(1-u) v(1-f))$, and $f(x ; r)$ describes the migration pattern. The expression of $f(x ; r)$ is $f(x ; r)=1 /(M-1)$ $(\cos (2 \pi x / M)+\cdots+\cos (2 \pi(M-1) x / M))$ if $r=\lceil M / 2\rceil$ $(\lceil x\rceil$ is an integer no greater than $x)$ and $f(x ; r)=1 / r$ $(\cos (2 \pi x / M)+\cdots+\cos (2 \pi r x / M))$ otherwise. When $r=$ $\lceil M / 2\rceil$, an individual can migrate to one of the other groups equiprobably, which is called global migration. Figure 5 shows when the intensity of selection is small, analytical results agree well with simulation results for different population sizes $(N$, Figures 5(a) and 5(b)), migration probabilities ( $v$, Figures 5(a) and 5(c)), mutation probabilities ( $u$, Figures 5(b) and 5(d)), group numbers $(M$, Figures $5(\mathrm{c})$ and $5(\mathrm{~d}))$, and migration patterns ( $r$, Figures 5(a) and 5(b)).

The mean offer increases with the mutation probability, the migration probability, or the group number (Figures 6(a), 6(b), and 6(d)) but decreases with the population size (Figure 6(c)). Accordingly, mutation, migration, or group structure promotes the evolution of fairness, but population size inhibits the evolution of fairness. The mean offer of $r=1$ is less than or equal to the one of global migration $(r=\lceil M / 2\rceil)$ or $r=2$. It implies that a smaller migration range leads to a fairer outcome than or the same 


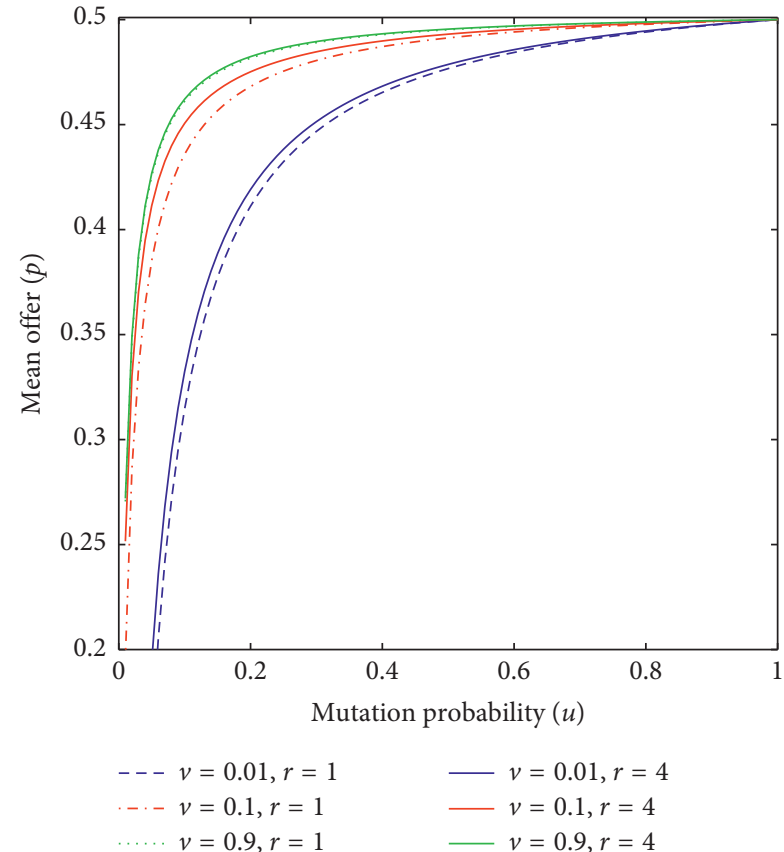

(a)

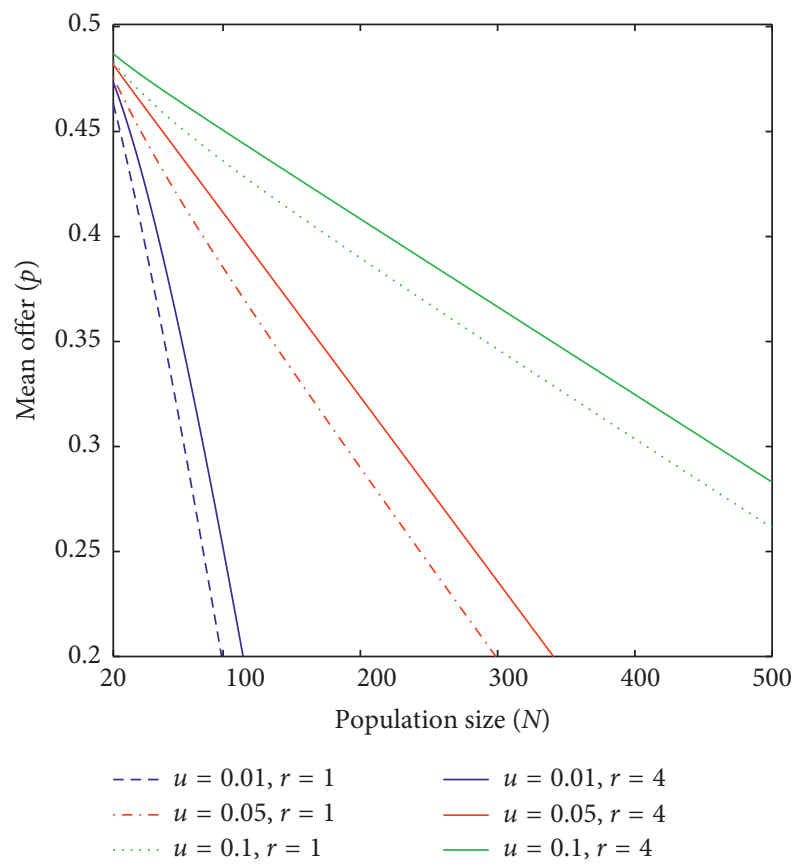

(c)

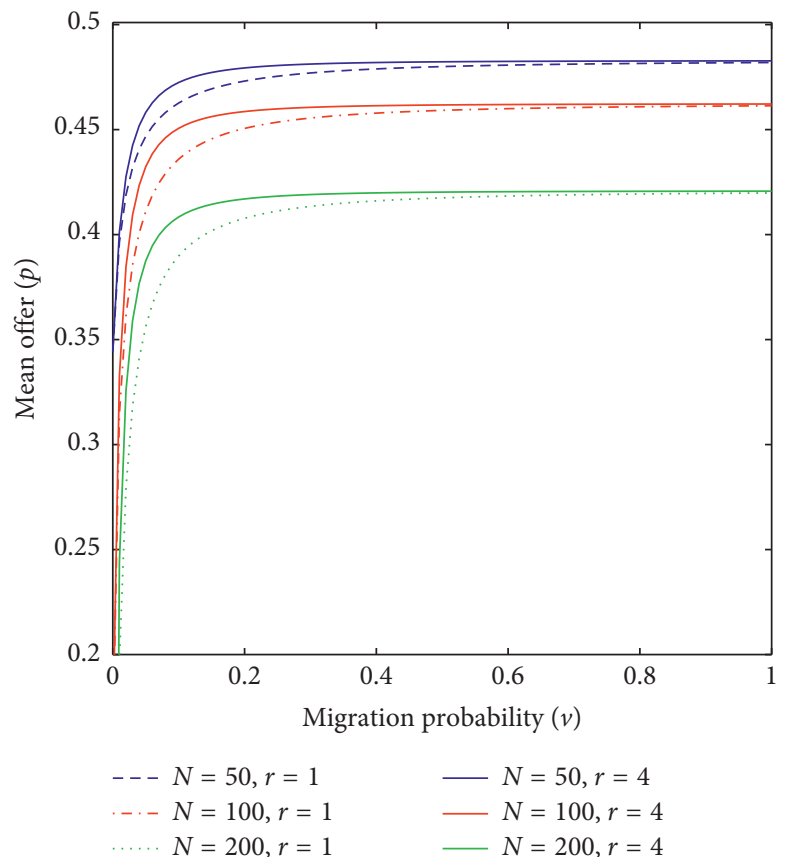

(b)

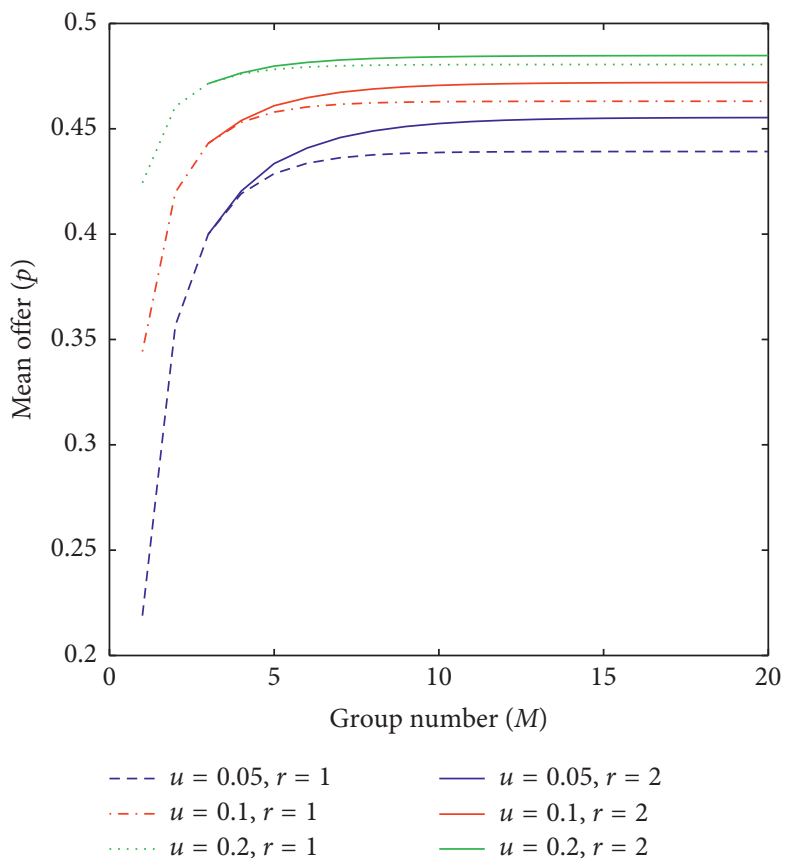

(d)

FIGURE 6: The effects of the mutation probability $u$, the migration probability $v$, the population size $N$, and the group number on the mean offer $p$ when all players use empathetic strategies $(\alpha=1)$. $p$ increases with $u$ in (a), increases with $v$ in (b), decreases with $N$ in (c), and increases with $M$ in (d). $p$ of $r=1$ is less than or equal to the one of global migration $r=4$ or $r=2$. Parameters: $\omega=0.01$ : (a) $N=100$ and $M=9$, (b) $u=0.1$ and $M=9$, (c) $v=0.1$ and $M=9$, and (d) $N=50$ and $v=0.1$.

outcome as a larger migration range. The result is tested against various population sizes, mutation probabilities, migration probabilities, and group numbers. Here, we focus on the mean offer of global migration, which can be obtained analytically.
From $f(x ;\lceil M / 2\rceil)=1 /(M-1) \sum_{j=1}^{M-1} \cos (2 \pi j x / M)$, we have $f(M ;\lceil M / 2\rceil)=1$ and $f(x ;\lceil M / 2\rceil)=-1 /(M-1)$ when $x \in\{1, \ldots, M-1\}$. Substituting these values into equation (2), we have 


$$
\begin{aligned}
p= & \frac{1}{2}-\omega \frac{(1-u)(N-1) N}{24 M} \\
& \cdot\left(\frac{1}{1+(N-1) u}+\frac{(M-1)(1-v(M / M-1))}{(1+(N-1) v(M / M-1))(1+(N-1) u+(N-1) v(M / M-1)-(N-1) u v(M / M-1))}\right) .
\end{aligned}
$$

It is easy to verify that the mean offer increases with the mutation probability, the migration probability, or the group number, but decreases with the population size. In order to make sure that almost all players can participate in games, we here assume that the population size is two times more than the group number $M$.

It has been shown that under strong selection, the mean offer and demand change significantly with the level of empathy $\alpha$. Moreover, the results for any level of empathy are between those under two extreme cases $\alpha=1$ and $\alpha=0$. Therefore, we calculate the approximate values of the mean offer and demand for $\alpha=1$ and $\alpha=0$. When $\alpha=1$, we use the replicator dynamics of $S$ discrete strategies $A=\{0,(1 / S-1), \ldots, 1\}$. By employing $x_{i}$ to denote the frequency of the strategy $i \in A$, we have

$$
\frac{d x_{i}}{d t}=\frac{x_{i} \times \pi_{i} \times(1-u)}{\phi}+\frac{u}{S}-x_{i},
$$

where $\pi_{i}$ is the payoff of the strategy $i$ and $\phi=\sum_{i=1}^{S} x_{i} \pi_{i}$ is the average payoff of the population. When $\alpha=0$, we use the replicator dynamics of $S^{2}$ discrete strategies. Similarly, by using $x_{(i, j)}$ to denote the frequency of the strategy $(i, j) \in A \times A$, we have

$$
\frac{d x_{(i, j)}}{d t}=\frac{x_{(i, j)} \times \pi_{(i, j)} \times(1-u)}{\Phi}+\frac{u}{S^{2}}-x_{(i, j)},
$$

where $\pi_{(i, j)}$ is the payoff of the strategy $(i, j)$ and $\Phi=\sum_{i=1}^{S} \sum_{j=1}^{S} x_{(i, j)} \pi_{(i, j)}$ is the average payoff of the population. When all strategies have identical initial frequencies, the stationary solutions of the above replicator equations, which no longer change with time, are denoted by $\bar{x}_{i}$ and $\bar{x}_{(i, j)}$. When $\alpha=1$, we approximate the mean offer by $\sum_{i=1}^{S}(i-1) /(S-1) \bar{x}_{i}$ with sufficiently large $S$. When $\alpha=0$, we approximate the mean offer and the mean demand by $\sum_{i=1}^{S}(i-1) /(S-1) \bar{x}_{(i, j)}$ and $\sum_{j=1}^{S}(j-1) /(S-1) \bar{x}_{(i, j)}$ with sufficiently large $S^{2}$, respectively. As shown in Figure 7, our approximations agree well with simulated results for different mutation probabilities.

\section{Discussion and Conclusions}

It has been shown that the mean offer typically ranges from 0.3 to 0.5 and the mean demand tends to lie between 0.2 and 0.35 in [23] by analyzing the data of many behavioral experiments. If the intensity of selection is very small or the mutation probability is high, the mean offer and demand cannot lie in the above ranges (closer to 0.5 ) because neutral drift or mutation dominates the evolutionary dynamics. At appropriate level of randomness and empathy, we quantitatively reproduce the above ranges. For very low-mutation probabilities and large intensities of selection (Figures 2(a), 2(b), 3(c), and 3(d)), intermediate empathy (the level of empathy $\alpha$ is intermediate) results in the experimental behaviors. Here, the combination of the mutation probability and the intensity of selection corresponds to low randomness. For very low-mutation probabilities and small intensities of selection (Figures 2(a), 2(b), 3(a), and 3(b)) or for intermediate-mutation probabilities and large intensities of selection (Figures 2(c), 2(d), 3(c), and 3(d)), small empathy ( $\alpha$ is small) leads to the experimental behaviors. Here, the combination of the mutation probability and the intensity of selection induces relatively high randomness. In all, low randomness together with intermediate empathy or relatively high randomness together with small empathy is sufficient to quantitatively reproduce the experimental behaviors.

One relevant literature [23] has reported that the average behavior of experiments can be quantitatively reproduced by adjusting the level of randomness in well-mixed populations. Our group structure itself cannot promote the evolution of fairness compared with well-mixed structure, and yet our group structure together with migration can. Besides the group structure, the largest differences between our model and their model are the update rule and the dependence of the fitness on the payoff, which lead to different results from the following two aspects. In their model, the level of randomness is continually decreased by continually increasing the intensity of selection, and so are the mean offer and demand. However, in our model, when the intensity of selection $\omega$ is sufficiently large, the level of randomness cannot be further decreased by increasing $\omega$ and neither are the mean offer and demand. The difference between their results and our results can be understood as follows. The pairwise comparison rule, which they adopted, implies that the payoff advantage of a lower offer over a higher offer can be increasingly enlarged by raising large $\omega$. The Moran process and the linear dependence of the fitness on the payoff, which we assume, make $\omega$ omitted from the numerator and the denominator of the probability that a player reproduces an offspring. It implies that sufficiently large intensities of selection no longer enlarge the payoff difference of one strategy over another.

Another relevant literature [20] introduced empathy by assuming that an individual offers his $q$-value with a given probability and has found that neither empathy nor fairness can be selected by natural selection. Our model allows interaction to only occur among players in the same group, so empathy can survive in the population according to [35] and fairness can further be selected. The previous literature [20] then studied another form of introducing empathy, where the mean offer $p$ and demand $q$ have been reported close to 0.5 . The result is clearly not in accordance with experimental 


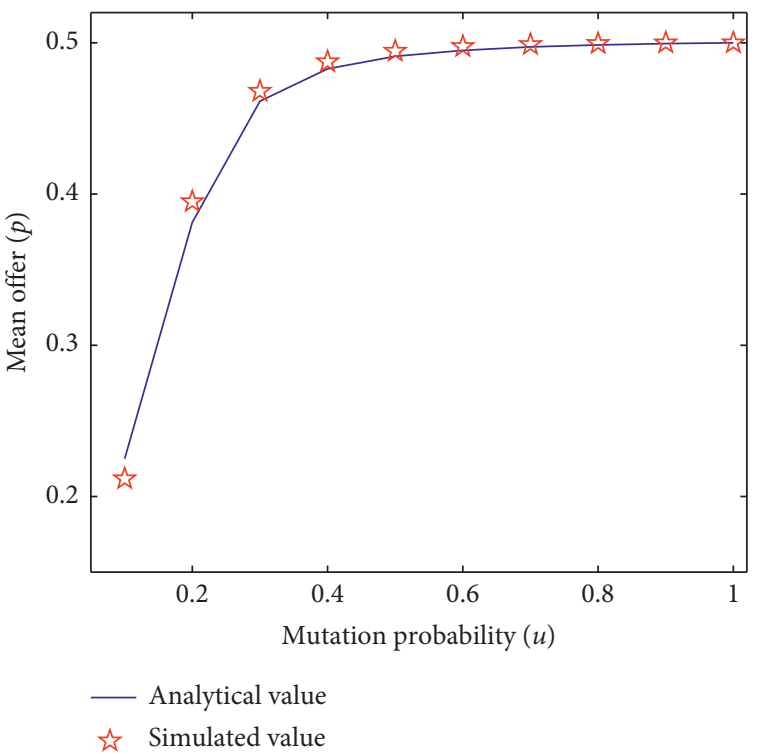

(a)

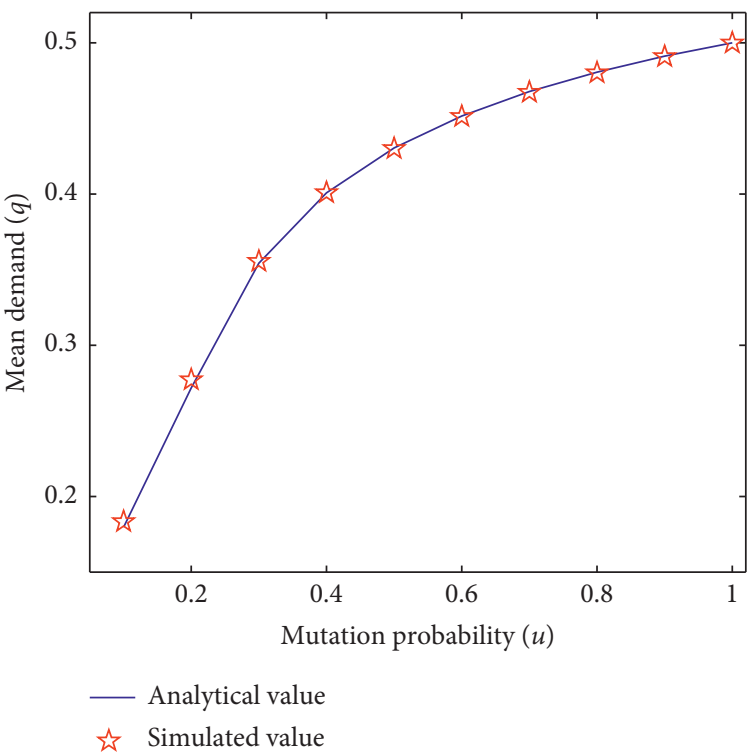

(b)

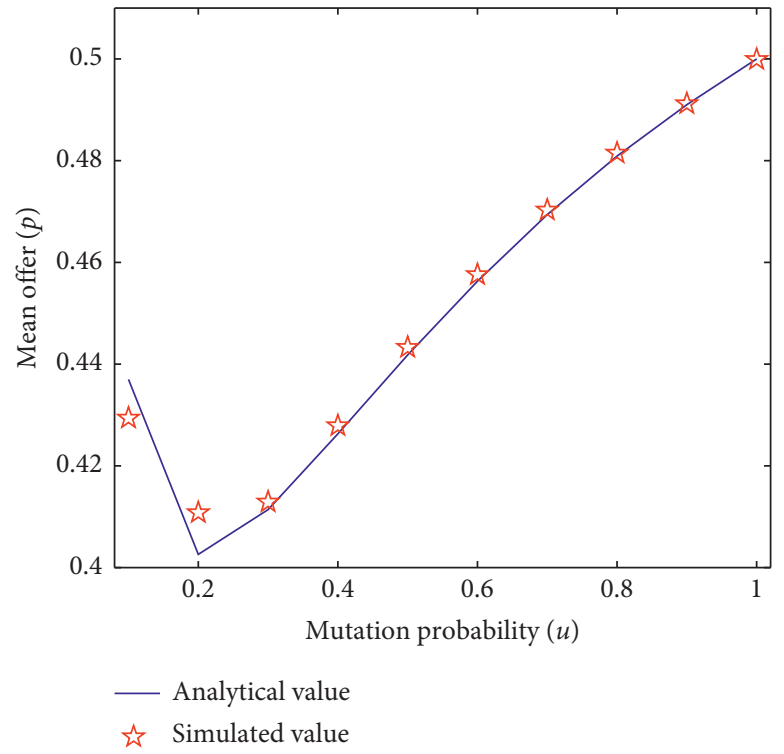

(c)

FiguRE 7: Under strong selection ( $\omega=1)$, comparison of theoretical results and numerical results. ( $\mathrm{a}, \mathrm{b})$ When all players use independent strategies $(\alpha=0)$, our analytical calculations of the mean offer $p$ and demand $q(S=7,30,40)$ are in good agreement with the simulated values for different mutation probabilities $u$. (c) When all players use empathetic strategies $(\alpha=1)$, our analytical calculations of $p(S=100)$ are in good agreement with the simulated values for different $u$.

evidence. The difference mainly depends on how strategy and empathy evolve. In their model, both strategy and empathy evolve by the modified adaptive dynamics, implying local mutation (i.e., mutants have to be close to the parent). In our model, both strategy and empathy evolve according to the mutation-selection process, and strategy is subject to global mutation (i.e., mutants can be quite different from the parent).

To sum up, we consider the effects of empathy on the evolution of fairness with global mutation and the intensity of selection. Different from the results reported in [20], where the mean offer and demand are both reported close to
0.5 , we quantitatively reproduce the experimental behaviors at low randomness with intermediate empathy or relatively high randomness with small empathy. We allow an arbitrary level of empathy in a mutation-selection process with group structure, different from the previous studies on empathy [18-20], where researchers have neglected the effects of mutation and the intensities of selection. We show that with low randomness, more empathy leads to a fairer outcome with a higher mean offer and demand. Counterintuitively, more empathy corresponds to a lower mean offer together with a higher mean demand for relatively high randomness. Finally, we analytically provide the mean offer and demand 
under both weak and strong intensities of selection when the largest or smallest level of empathy is introduced. We find that for the largest level of empathy together with weak selection, mutation, migration, or group structure can promote the evolution of fairness, while population size cannot. Previous theoretical studies of fairness were mainly conducted with agent-based simulations, where only few presented analytical results (but with strong assumptions).

\section{Data Availability}

The data used to support the findings of this study are available from the corresponding author upon request.

\section{Conflicts of Interest}

The authors declare that there are no conflicts of interest regarding the publication of this paper.

\section{Authors' Contributions}

Y. Z. and J. L. contributed equally to this work. Y. Z. and A. L. designed the project. All authors performed the research. Y. Z. performed the theoretical calculations. J. L. performed the numerical simulations. A. L. and Y. Z. analyzed the results and wrote the manuscript, and J. L. edited the manuscript.

\section{Acknowledgments}

Y. Z. is grateful for the support by the National Natural Science Foundation of China (grant nos. 61603036, 61520106009 and 61533008) and the Fundamental Research Funds for the Central Universities (grant no. FRF-TP-18036A2). A. L. acknowledges the International Human Frontier Science Program Postdoctoral Fellowship (grant no. LT000696/2018-C) and the support from Foster Lab at University of Oxford.

\section{Supplementary Materials}

Calculation of the mean offer $p$ in the main text for the empathetic strategies. Table 1: for different levels of empathy $a$, the average values and the standard deviations of the mean offer $p$ and the mean demand $q$. For each set of parameters, we perform 10 simulations generated by different random seeds. Each simulation runs $5 \times(10)^{7}$ generations. Other parameters: $N=50, M=9, r=1, u=0.2, v=0.1$, and $\omega=1$. Table 2: for different probability density distributions of initialization $f(x)$, the mean offer $p$ and the mean demand $q$. For each set of parameters, we perform one simulation with $5 \times(10)^{7}$ generations. Other parameters: $N=50, M=9, r=1$, $u=0.01, v=0.1$, and $\omega=1$. Figure S1: the changing trends of the mean offer $p$ and demand $q$ with the level of empathy $a$ or the intensity of selection $\omega$. ( $\mathrm{a}$ and b) $p$ and $q$ change very little with $a$ when $\omega$ is very small. (c and $d$ ) When the mutation probability is high, the experimental behaviors cannot be observed. Parameters: $N=50, M=9, r=1$, and $v=0.1$, with $u=0.1$ in $(\mathrm{a}, \mathrm{b})$ and $u=0.4$ in (c, d). Figure S2 : the mean offer $p$ and demand $q$ for the smallest level of empathy $a=0$. (a, b) When only the offspring migrates at a generation, $p$ and $q$ increase with small $r$ but remain around constants when $r$ is large; when all individuals migrate at a generation, $p$ and $q$ do not significantly change with $r$. (c, d) When there is no migration (the migration probability $v=0$ ), $p$ and $q$ do not change with the number of groups $M$. Parameters: $N=50$ : (a, b) $\omega=0.015, M=16$, $v=0.1$; (c, d) $\omega=0.01, v=0$, and $r=1$. (Supplementary Materials)

\section{References}

[1] C. F. Camerer, Behavioral Game Theory: Experiments in Strategic Interaction, Princeton University Press, Princeton, NJ, USA, 2011.

[2] W. Güth and M. G. Kocher, "More than thirty years of ultimatum bargaining experiments: motives, variations, and a survey of the recent literature," Journal of Economic Behavior \& Organization, vol. 108, pp. 396-409, 2014.

[3] W. Güth, R. Schmittberger, and B. Schwarze, "An experimental analysis of ultimatum bargaining," Journal of Economic Behavior \& Organization, vol. 3, no. 4, pp. 367-388, 1982.

[4] X. Han, S. Cao, J. Bao et al., "Equal status in ultimatum games promotes rational sharing," Scientific Reports, vol. 8, p. 1222, 2018.

[5] S. Debove, N. Baumard, and J.-B. André, "Models of the evolution of fairness in the ultimatum game: a review and classification," Evolution and Human Behavior, vol. 37, no. 3, pp. 245-254, 2016.

[6] A. Szolnoki and X. Chen, "Reciprocity-based cooperative phalanx maintained by overconfident players," Physical Review E, vol. 98, no. 2, Article ID 022309, 2018.

[7] M. Perc, J. J. Jordan, D. G. Rand, Z. Wang, S. Boccaletti, and A. Szolnoki, "Statistical physics of human cooperation," Physics Reports, vol. 687, pp. 1-51, 2017.

[8] M. Perc and A. Szolnoki, "A double-edged sword: benefits and pitfalls of heterogeneous punishment in evolutionary inspection games," Scientific Reports, vol. 5, p. 11027, 2015.

[9] A. Szolnoki and M. Perc, "Second-order free-riding on antisocial punishment restores the effectiveness of prosocial punishment," Physical Review X, vol. 7, no. 4, Article ID 041027, 2017.

[10] A. Li and L. Wang, "Evolutionary dynamics of synergistic and discounted group interactions in structured populations," Journal of Theoretical Biology, vol. 377, pp. 57-65, 2015.

[11] Q. Su, A. Li, L. Zhou, and L. Wang, "Interactive diversity promotes the evolution of cooperation in structured populations," New Journal of Physics, vol. 18, no. 10, Article ID 103007, 2016.

[12] F. Fu and M. A. Nowak, "Global migration can lead to stronger spatial selection than local migration," Journal of Statistical Physics, vol. 151, no. 3-4, pp. 637-653, 2013.

[13] A. Liu, Y. Zhang, X. Chen, and C. Sun, "Randomness and diversity matter in the maintenance of the public resources," EPL (Europhysics Letters), vol. 117, no. 5, p. 58002, 2017.

[14] Q. Su, A. Li, and L. Wang, "Evolutionary dynamics under interactive diversity," New Journal of Physics, vol. 19, no. 10, p. 103023, 2017.

[15] M. A. Nowak, K. M. Page, and K. Sigmund, "Fairness versus reason in the ultimatum game," Science, vol. 289, no. 5485, pp. 1773-1775, 2000.

[16] K. M. Page and M. A. Nowak, "A generalized adaptive dynamics framework can describe the evolutionary ultimatum 
game," Journal of Theoretical Biology, vol. 209, no. 2, pp. 173-179, 2001.

[17] A. Sánchez and J. A. Cuesta, "Altruism may arise from individual selection," Journal of Theoretical Biology, vol. 235, no. 2, pp. 233-240, 2005.

[18] A. Szolnoki, M. Perc, and G. Szabó, "Defense mechanisms of empathetic players in the spatial ultimatum game," Physical Review Letters, vol. 109, no. 7, Article ID 078701, 2012.

[19] R. Sinatra, J. Iranzo, J. Gómez-Gardeñes, L. M. Floría, V. Latora, and Y. Moreno, "The ultimatum game in complex networks," Journal of Statistical Mechanics: Theory and Experiment, vol. 2009, no. 9, Article ID P09012, 2009.

[20] K. Page and M. A. Nowak, "Empathy leads to fairness," Bulletin of Mathematical Biology, vol. 64, no. 6, pp. 1101-1116, 2002.

[21] T. Wu, F. Fu, Y. Zhang, and L. Wang, "Adaptive tag switching reinforces the coevolution of contingent cooperation and tag diversity," Journal of Theoretical Biology, vol. 330, pp. 45-55, 2013.

[22] Z. Yang, Z. Li, T. Wu, and L. Wang, "Effects of partner choice and role assignation in the spatial ultimatum game," EPL (Europhysics Letters), vol. 109, no. 4, Article ID 40013, 2015.

[23] D. G. Rand, C. E. Tarnita, H. Ohtsuki, and M. A. Nowak, "Evolution of fairness in the one-shot anonymous ultimatum game," Proceedings of the National Academy of Sciences, vol. 110, no. 7, pp. 2581-2586, 2013.

[24] X. Wang, X. Chen, and L. Wang, "Random allocation of pies promotes the evolution of fairness in the ultimatum game," Scientific Reports, vol. 4, p. 4534, 2014.

[25] P. Forber and R. Smead, "The evolution of fairness through spite," Proceedings of the Royal Society B: Biological Sciences, vol. 281, no. 1780, Article ID 20132439, 2014.

[26] Y. Zhang and F. Fu, "Strategy intervention for the evolution of fairness," PLoS One, vol. 13, no. 5, Article ID e0196524, 2018.

[27] Y. Zhang, X. Chen, A. Liu, and C. Sun, "The effect of the stake size on the evolution of fairness," Applied Mathematics and Computation, vol. 321, pp. 641-653, 2018.

[28] A. Szolnoki, M. Perc, and G. Szabó, "Accuracy in strategy imitations promotes the evolution of fairness in the spatial ultimatum game," EPL (Europhysics Letters), vol. 100, no. 2, p. 28005, 2012.

[29] K. M. Page, M. A. Nowak, and K. Sigmund, "The spatial ultimatum game," Proceedings of the Royal Society of London. Series B: Biological Sciences, vol. 267, no. 1458, pp. 2177-2182, 2000.

[30] T. Killingback and E. Studer, "Spatial ultimatum games, collaborations and the evolution of fairness," Proceedings of the Royal Society of London. Series B: Biological Sciences, vol. 268, no. 1478, pp. 1797-1801, 2001.

[31] M. N. Kuperman and S. Risau-Gusman, "The effect of the topology on the spatial ultimatum game," The European Physical Journal B, vol. 62, no. 2, pp. 233-238, 2008.

[32] J. Gao, Z. Li, T. Wu, and L. Wang, "The coevolutionary ultimatum game," EPL (Europhysics Letters), vol. 93, no. 4, Article ID 48003, 2011.

[33] J. Iranzo, J. Román, and A. Sánchez, “The spatial ultimatum game revisited," Journal of Theoretical Biology, vol. 278, no. 1, pp. 1-10, 2011.

[34] X. Wang, X. Chen, and L. Wang, "Evolutionary dynamics of fairness on graphs with migration," Journal of Theoretical Biology, vol. 380, pp. 103-114, 2015.

[35] J. Iranzo, L. M. Floria, Y. Moreno, and A. Sanchez, "Empathy emerges spontaneously in the ultimatum game: small groups and networks," PLoS One, vol. 7, no. 9, Article ID e43781, 2012.

[36] A. Li, M. Broom, J. Du, and L. Wang, "Evolutionary dynamics of general group interactions in structured populations," Physical Review E, vol. 93, no. 2, Article ID 022407, 2016.

[37] F. P. Santos, F. C. Santos, A. Paiva, and J. M. Pacheco, "Evolutionary dynamics of group fairness," Journal of Theoretical Biology, vol. 378, pp. 96-102, 2015.

[38] D. Helbing and W. Yu, "The outbreak of cooperation among success-driven individuals under noisy conditions," Proceedings of the National Academy of Sciences, vol. 106, no. 10, pp. 3680-3685, 2009.

[39] A. Li, S. P. Cornelius, Y.-Y. Liu, L. Wang, and A.-L. Barabási, "The fundamental advantages of temporal networks," Science, vol. 358, no. 6366, pp. 1042-1046, 2017.

[40] F. A. Rodrigues, T. K. D. Peron, P. Ji, and J. Kurths, "The kuramoto model in complex networks," Physics Reports, vol. 610, pp. 1-98, 2016.

[41] S. Saavedra, F. Reed-Tsochas, and B. Uzzi, "A simple model of bipartite cooperation for ecological and organizational networks," Nature, vol. 457, no. 7228, pp. 463-466, 2009.

[42] N. Masuda, S. Kojaku, and Y. Sano, "Configuration model for correlation matrices preserving the node strength," Physical Review E, vol. 98, no. 1, Article ID 012312, 2018.

[43] I. Zähle, J. T. Cox, and R. Durrett, "The stepping stone model. ii: genealogies and the infinite sites model," The Annals of Applied Probability, vol. 15, no. 1B, pp. 671-699, 2005.

[44] T. Uehara and Y. Iwasa, "Global mutations and local mutations have very different effects on evolution, illustrated by mixed strategies of asymmetric binary games," Journal of Theoretical Biology, vol. 262, no. 2, pp. 223-231, 2010.

[45] A. Traulsen, C. Hauert, H. De Silva, M. A. Nowak, and K. Sigmund, "Exploration dynamics in evolutionary games," Proceedings of the National Academy of Sciences, vol. 106, no. 3, pp. 709-712, 2009.

[46] A. Traulsen, D. Semmann, R. D. Sommerfeld, H.-J. Krambeck, and M. Milinski, "Human strategy updating in evolutionary games," Proceedings of the National Academy of Sciences, vol. 107, no. 7, pp. 2962-2966, 2010.

[47] B. Allen, A. Traulsen, C. E. Tarnita, and M. A. Nowak, "How mutation affects evolutionary games on graphs," Journal of Theoretical Biology, vol. 299, pp. 97-105, 2012.

[48] M. A. Nowak, A. Sasaki, C. Taylor, and D. Fudenberg, "Emergence of cooperation and evolutionary stability in finite populations," Nature, vol. 428, no. 6983, pp. 646-650, 2004.

[49] T. Antal, H. Ohtsuki, J. Wakeley, P. D. Taylor, and M. A. Nowak, "Evolution of cooperation by phenotypic similarity," Proceedings of the National Academy of Sciences, vol. 106, no. 21, pp. 8597-8600, 2009.

[50] C. E. Tarnita, T. Antal, H. Ohtsuki, and M. A. Nowak, "Evolutionary dynamics in set structured populations," Proceedings of the National Academy of Sciences, vol. 106, no. 21, pp. 8601-8604, 2009.

[51] C. E. Tarnita, N. Wage, and M. A. Nowak, "Multiple strategies in structured populations," Proceedings of the National Academy of Sciences, vol. 108, no. 6, pp. 2334-2337, 2011.

[52] F. A. Matsen and J. Wakeley, "Convergence to the islandmodel coalescent process in populations with restricted migration," Genetics, vol. 172, no. 1, pp. 701-708, 2006. 


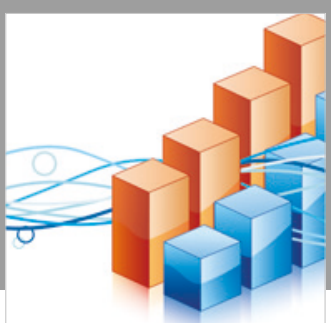

Advances in

Operations Research

\section{-n-m}
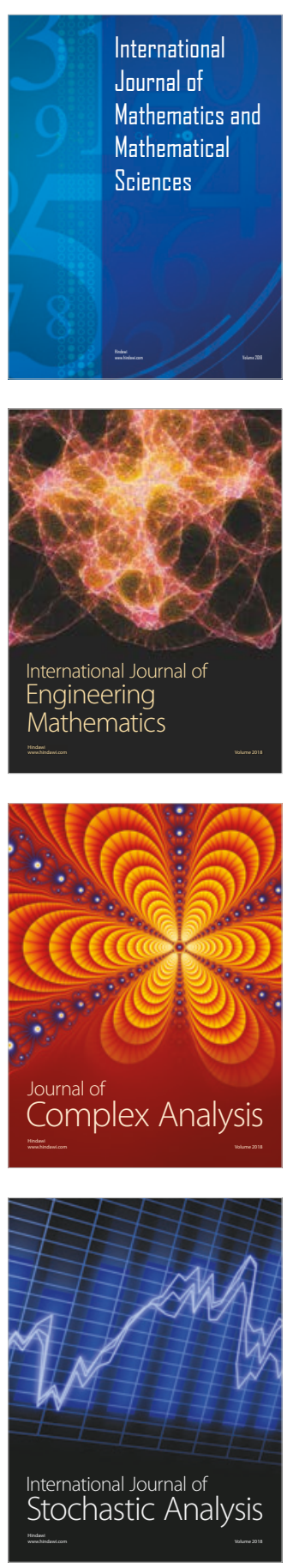
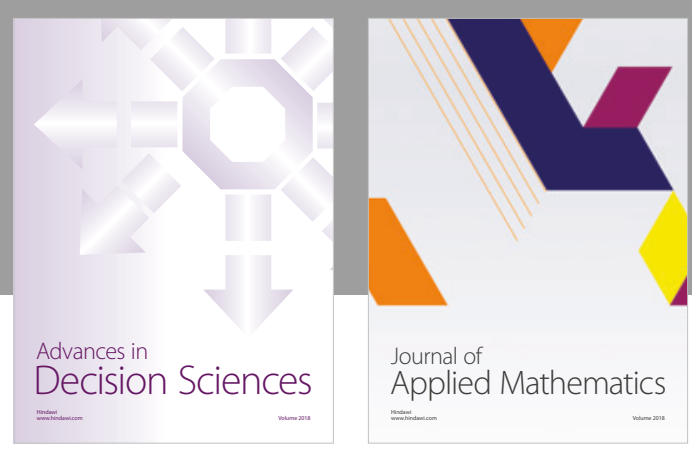

Journal of

Applied Mathematics
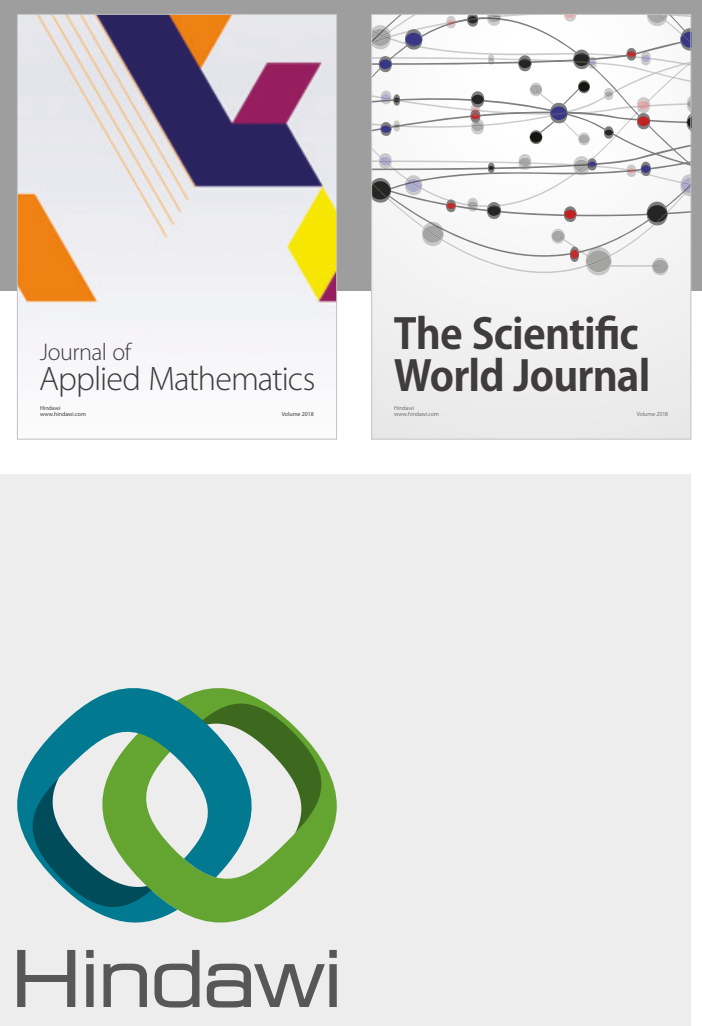

Submit your manuscripts at

www.hindawi.com

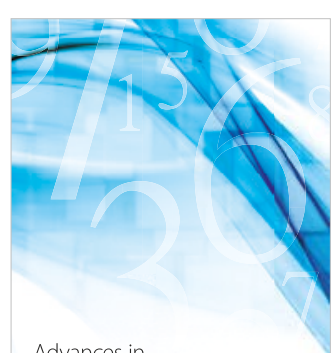

Advances in
Numerical Analysis
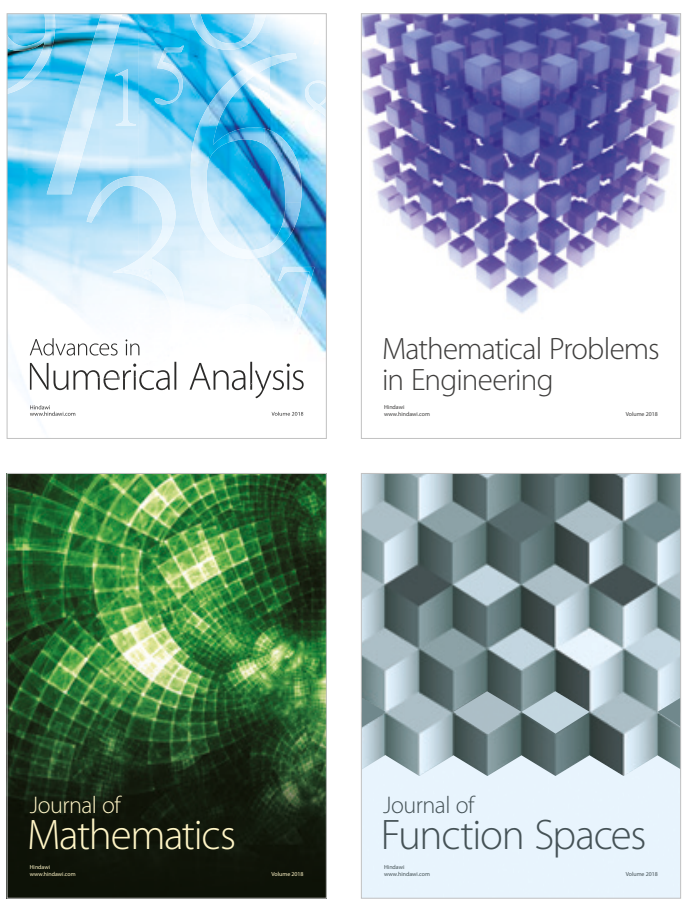

Mathematical Problems in Engineering

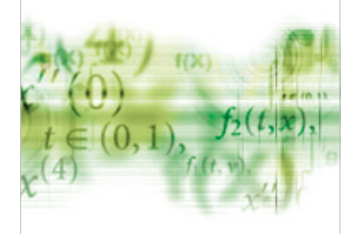

International Journal of

Differential Equations

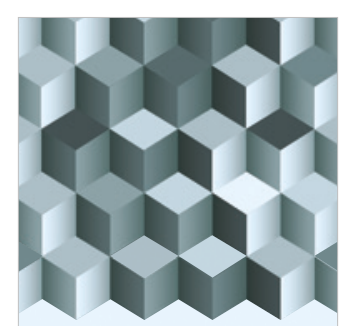

Journal of

Function Spaces
The Scientific

World Journal

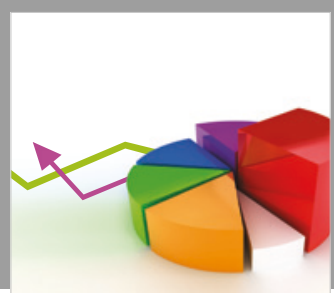

Journal of

Probability and Statistics
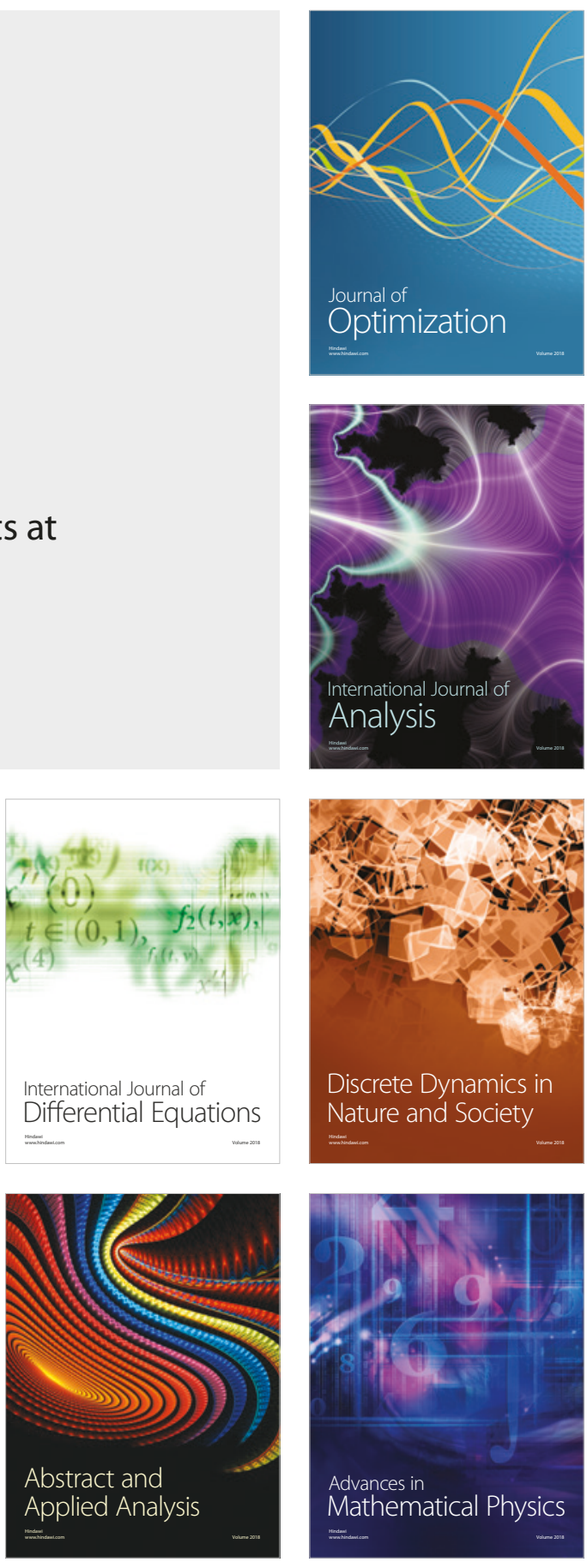